\title{
Role of bronchoalveolar lavage in evaluating new pulmonary infiltrates on computed tomography in haematology patients with fever unresponsive to broad-spectrum antibiotics
}

Pulmonary complications are a significant cause of morbidity and mortality in immunocompromised haemato-oncology patients, with invasive fungal infection being one of the commonest. Chest computed tomography (CT) with bronchoalveolar lavage (BAL) for further investigation of new pulmonary changes identified on CT is frequently used to investigate patients with neutropenic fever unresponsive to broad-spectrum antibiotics. However, studies of BAL are conflicting, with diagnostic yields of 15$80 \%$ (Milburn et al., 1987; Saito et al., 1988; Peikert et al., 2005), whilst its role in diagnosing non-infectious aetiologies is limited. Complication rates approach $15 \%$ in haematology patients and some studies have failed to demonstrate a beneficial impact on patient outcome in response to BAL findings (White et al., 1997; Murray et al., 2001). The optimum role of BAL in the diagnostic pathway of this high-risk population remains unclear.

We attempted to establish the diagnostic yield, complication rate and impact on therapy and outcome of BAL in neutropenic haematology patients with fever unresponsive to broad-spectrum antibiotics and new pulmonary changes on CT.

Addenbrooke's Hospital is a tertiary referral hospital providing chemotherapy and stem cell transplantation for haematology patients. All haematology inpatients undergoing CT chest for antibiotic-unresponsive neutropenic fever (neutrophil count $<0.5 \times 10^{9} 1^{-1}$ ) between September 2003 and December 2006 were retrospectively identified from a radiology database. Radiology findings,

histopathology and admission records were reviewed and patients undergoing BAL were identified. All BAL samples growing fungi in the study period were also analysed. Specimen processing from BAL was audited against British Society for
Medical Mycology (BSMM) guidelines (Denning et al., 2003). These state that all bronchoscopy fluids from patients suspected of infection should be examined microscopically for hyphae and cultured on specialized media, all isolates should be identified to species level and all tissues from immunocompromised patients with suspected infection should be stained with fungal stains. BAL samples were also tested for routine microbiology, mycobacteria, Pneumocystis and viruses. Fungal antigen and nucleic acid amplification tests were not available during the study period.

Death and admission duration data were collected for all patients with new pulmonary changes on CT. Approval was obtained from the clinical audit department.

During the study period, 191 patients (198 episodes) underwent a chest CT scan of which 68 scans demonstrated significant new radiological changes. Sixty of these episodes came from 242 patients who received intensive chemotherapy for acute leukaemia or myelodysplasia and an additional 260 patients who underwent stem cell transplantation (179 autologous, 81 allogeneic) during the study period. The remaining eight patients were drawn from an unknown total number of patients receiving other therapies in the department during the study period. The median age of the 68 patients was 33 years (range 1-68; 17 were $<16$ years) and $35(51 \%)$ were male. Diagnoses are summarized in Table 1. All patients had neutropenic fever at the time of commencing antibiotics. The median duration of neutropenia prior to starting antibiotics was 11 days (range 269). By the time of CT scan, the median neutrophil count was $0.2 \times 10^{9} 1^{-1}$ and 20 patients had a neutrophil count $>1.0 \times 10^{9}$ $1^{-1}$. All 68 patients had received $\geqslant 3$ days of antibiotics (usually piperacillintazobactam, vancomycin and gentamicin in combination) and had commenced antifungal therapy [liposomal amphotericin B $(n=66)$ or voriconazole $(n=2)$ ] by the time that the CT scan was performed. Chest CT was performed within $24 \mathrm{~h}$ of commencing systemic antifungal therapy in $93 \%$ of cases. Bronchoscopy was performed in 24 cases (BAL in 24 cases, biopsy in three cases). The median time from CT scan to bronchoscopy was 7 days (range -6 to 12 days; the negative figure reflects two patients who had bronchoscopy and BAL shortly after commencing systemic antifungal therapy 6 and 4 days before a CT scan was performed because of poor clinical status and severely abnormal plain chest radiograph). The median platelet count at the time of bronchoscopy was $39 \times 10^{9} 1^{-1}$ (range 15-559 $\times 10^{9} 1^{-1}$ ) and most patients were considered unsuitable for biopsy. Microbiology received a sample in $21(87.5 \%)$ cases and cytology in 12 (50\%). The sample was unavailable/ unsuitable for analysis in two (8\%) cases. Culture of BAL fluid was positive in two cases (Klebsiella pneumoniae and Pseudomonas aeruginosa; both patients bacteraemic) and PCR for herpes simplex was positive in one patient. BSMM audit standards for microbiology (95\%), cytology (92\%) and histopathology (100\%) audit requirements were largely met. No non-infective diagnoses were established. The length of stay after CT was longer in patients who underwent $\mathrm{BAL}$ (median 35 days versus 21 days) and mortality was higher in those undergoing BAL (21\% versus $7 \%$ ) though neither difference achieved statistical significance ( $P>0.05$, Pearson's chi-square test). Three patients $(12.5 \%)$ developed respiratory distress, one of whom required ventilatory support.

Although a large recent study has supported the role of BAL in the assessment of such patients, it did not assess the clinical benefit following BAL or 
Table 1. Disease characteristics of affected patients

\begin{tabular}{|lc|}
\hline Disease & Number \\
\hline Acute myeloid leukaemia & 32 \\
Acute lymphoblastic leukaemia & 12 \\
Allogeneic stem cell transplant & 12 \\
Autologous stem cell transplant & 4 \\
Non-Hodgkin's lymphoma & 4 \\
Other* & 4 \\
Total & 68 \\
\hline
\end{tabular}

${ }^{\star}$ One each of chronic myeloid leukaemia, chronic lymphocytic leukaemia, myeloma and aplastic anaemia.

report on complications other than death (Hummel et al., 2008). It also failed to state the type of antimicrobial agents used in the study, and these may have changed over the 15-year study period. Our data, although limited, suggest than in patients empirically treated with broad-spectrum antibiotic and antifungal therapy, BAL has little value and, in accord with many other studies, is a procedure with a significant complication rate. Whilst this study is limited by its single centre retrospective design, the cohort examined nonetheless represents a typical stem-cell transplant centre with many patients with neutropenia (profound and prolonged) and thrombocytopenia. Another potential criticism of the study is that most patients were sampled in the early risk period for invasive mould infections in haematology patients, which may have missed later cases of fungal disease. We reviewed all microbiology records that grew moulds in the study period to counter this. The diagnostic yield of BAL was low, despite national guidelines being largely followed, and the results only changed treatment for one patient. In addition, three patients developed complications, one potentially life-threatening. BAL did not seem to improve patient outcome or reduce duration of admission.

Our study raises questions about the validity of the widely adopted practice of BAL for neutropenic patients with CT changes and fever unresponsive to broadspectrum antibiotics. Our data suggest that it may be more appropriate to consider either excluding BAL or delaying it until antifungal therapy proved ineffective. Future studies should also explore the possible role of fungal antigen detection techniques in supplanting the need for, or refining the indications for, BAL.

\section{T. Todd ${ }^{1}$ and D. A. Enoch ${ }^{2} \dagger$}

${ }^{1}$ Department of Haematology, Addenbrooke's Hospital, Hills Road, Cambridge CB2 2OW, UK

${ }^{2}$ Clinical Microbiology \& Public Health Laboratory, Addenbrooke's Hospital, Hills Road, Cambridge CB2 20W, UK

Correspondence: D. A. Enoch (David.enoch@pbh-tr.nhs.uk)
†Present address: Clinical Microbiology \& Public Health Laboratory, Peterborough District Hospital, Thorpe Road, Peterborough PE3 6JW, UK.

Denning, D. W., Kibbler, C. C., Barnes, R. A. \& British Society for Medical Mycology (2003). British Society for Medical Mycology proposed standards of care for patients with invasive fungal infections. Lancet Infect Dis 3, 230-240.

Hummel, M., Rudert, S., Hof, H., Hehlmann, R. \& Buchheidt, D. (2008). Diagnostic yield of bronchoscopy with bronchoalveolar lavage in febrile patients with hematologic malignancies and pulmonary infiltrates. Ann Hematol 87, 291-297.

Milburn, H. J., Prentice, H. G. \& du Bois, R. M. (1987). Role of bronchoalveolar lavage in the evaluation of interstitial pneumonitis in recipients of bone marrow transplants. Thorax 42, 766-772.

Murray, P. V., O'Brien, M. E. R., Padhani, A. R., Powles, R., Cunningham, D., Jeanes, A. \& Ashley, S. (2001). Use of first line bronchoalveolar lavage in the immunosuppressed oncology patient. Bone Marrow Transplant 27, 967-971.

Peikert, T., Rana, S. \& Edell, E. S. (2005). Safety, diagnostic yield, and therapeutic implications of flexible bronchoscopy in patients with febrile neutropenia and pulmonary infiltrates. Mayo Clin Proc 80, 1414-1420.

Saito, H., Anaissie, E. J., Morice, R. C., Dekmezian, R. \& Bodey, G. P. (1988).

Bronchoalveolar lavage in the diagnosis of pulmonary infiltrates in patients with acute leukemia. Chest 94, 745-749.

White, P., Bonacum, J. T. \& Miller, C. B. (1997). Utility of fiberoptic bronchoscopy in bone marrow transplant patients. Bone Marrow Transplant 20, 681-687. 